\title{
Cosmological Model-independent Gamma-ray Bursts Calibration and its Cosmological Constraint to Dark Energy
}

\section{Lixin $\mathbf{X u}$}

Institute of Theoretical Physics, School of Physics \& Optoelectronic Technology, Dalian University of Technology, Dalian, 116024, P. R. China

College of Advanced Science \& Technology, Dalian University of Technology, Dalian, 116024, P. R. China

Korea Astronomy and Space Science Institute, Yuseong Daedeokdaero 776, Daejeon 305348 , R. Korea

E-mail: lxxu@dlut.edu.cn

\begin{abstract}
As so far, the redshift of Gamma-ray bursts (GRBs) can extend to $z \sim 8$ which makes it as a complementary probe of dark energy to supernova Ia (SN Ia). However, the calibration of GRBs is still a big challenge when they are used to constrain cosmological models. Though, the absolute magnitude of GRBs is still unknown, the slopes of GRBs correlations can be used as a useful constraint to dark energy in a completely cosmological model independent way. In this paper, we follow Wang's model-independent distance measurement method and calculate their values by using 109 GRBs events via the so-called Amati relation. Then, we use the obtained model-independent distances to constrain $\Lambda$ CDM model as an example.
\end{abstract}

ArXiv ePrint: 1005.5055 


\section{Contents}

1 Introduction 1

2 Calibration of GRBs and Model-independent Distance Measurement 2

3 Cosmological Constraint to Dark Energy $\quad 8$

4 Summary and Discussion $\quad 9$

A Cosmic Observations: SN, BAO and CMB $\quad 10$

A.1 Type Ia Supernovae constraints 10

A.2 Baryon Acoustic Oscillation constraints 11

$\begin{array}{lll}\text { A.3 Cosmic Microwave Background constraints } & 13\end{array}$

\section{Introduction}

Since the discovery of an accelerated expansion of our Universe through the observations of supernova Ia (SN Ia) [1, 2], many cosmic observations have been used to explore the nature of dark energy which has negative pressure and pushes the Universe into an accelerated phase. Particularly, the redshift of Gamma-ray burst (GRBs) can extend to higher redshift $z \sim 8$. This merit makes it as a complementary cosmic probe to SN Ia. However, a big challenge, the so-called circular problem, has to be overcome when one calibrates the GRBs to obtain the distances at different redshifts. In [3], Schaefer et. al. have derived the distance modulus in $\Lambda \mathrm{CDM}$ model by using five GRBs correlations. The so-called circular problem would be committed when one uses the resulted distance redshift relation to constrain any other cosmological models beyond $\Lambda \mathrm{CDM}$. To overcome this circular problem, Li, et. al [4] put the GRBs correlation and cosmological model constraint together. Via the Markov Chain Monte Carlo (MCMC) method, they fixed the calibration parameters and constrained the model parameter space simultaneously. However, it looks like using a 'ruler' having no marks to measure the length of an object. And after the measurement, the length and scale of ruler are given together. So the lack of calibration makes the GRBs lose the power to constrain cosmological models. Cosmography method was considered in [5] by parameterizing the luminosity distance $d_{L}$ in terms of deceleration $q_{0}$, jerk $j_{0}$ and snap $s_{0}$ parameters. In this way, the cosmological model dependent problem was removed. Liang et. al. [6] calibrated GRBs by using low redshift SN Ia and obtained a tight constrain to the cosmological model parameter space. This method was reconsidered by Wei $[7,8]$. By analyzing the calibration process carefully, one can find the potential drawback. The calibrated GRBs correlation makes GRBs have the same luminosity distance-redshifts relation as SN Ia at lower redshifts. In this way, the relation is extended to higher redshifts. Equivalently, one just extends the luminosity distance-redshifts relation of SN Ia to higher redshifts. So, it makes the obtained luminosity distance-redshifts relation strongly depends on that of SN Ia. The worst thing is that it makes the data points of GRBs useless, because one has known the luminosity distance-redshifts relation at high redshifts from SN Ia, though no SN Ia is found at the high redshift regions of GRBs. Alternatively, Wang presented a model-independent distance measurement from GRBs calibrated internally [18]. The main point of Wang's method is 
that the statistical errors of correlation parameters $\sigma_{a}, \sigma_{b}$ and systematic error $\sigma_{\text {sys }}$ obtained in $\Lambda$ CDM models are used, but are not the correlation parameters $a$ and $b$ themselves, for the definitions of $a, b$ please see Eq. (2.2). The viability of this implement comes from the observations that the errors of correlation parameters are almost the same for different values of $\Omega_{m 0}$ for $\Lambda \mathrm{CDM}$ model, though the values of $a$ and $b$ are really different. Then, in terms of a set of model-independent distance measurements, the cosmic constraint from GRBs is set up via cubic spline interpolation from cosmological model independent distance ratio $\bar{r}_{p}\left(z_{i}\right)$. The merits of this method are follows: (i) the constraint from GRBs is in a cosmological model independent way. It alleviates the circular problem. So, it can be used to constrain any other cosmological models. (ii) It is not calibrated by any other external data sets. It does not suffer any consistent problem when it is combined with other data sets as cosmic constraints. (iii) The cosmological model independent calibration is done firstly. It means that the 'ruler' has been marked. (iv) Though the absolute magnitude of GRBs is unkown, the slopes of GRBs correlations can be used as cosmological constraints.

Recently, Wei [8] used 109 GRBs data points via Amati relation [11-13] calibrated by SN Ia to constrain cosmological model. Based on the points mentioned above, to alleviate the data sets dependence and circular problem, we shall present our calculation results based on Wang's method via Amati correlation. In fact, we find a new $\chi_{G R B}^{2}$ which only depends on the slopes of GRBs correlation and make the absolute magnitude of GRBs irrelevant. For the details, please see section 2. As results, a set of model-independent distance measurements are obtained on the basis of Amati relation that can be used to constrain cosmological models.

However we have to stress that the Amati relation has been criticized for many reasons in the literatures. Li demonstrated an ambiguity in determining the redshifts of GRBs [14]. Of course, this ambiguity can be overcome when the redshift is well determined. The major criticism came from Nakar and Prian [15] who developed a test for the Amati relation even in the case where the redshifts of GRBs were unknown. The test was also generalized by Band and Preece [16]. They concluded that the Amati relation suffered the problem of selection bias. Recently, the authors of [17] have also concluded that the Amati relation is an artifact of selection effects with the burst population and the detector. They also point out that the Amati relation is failed whether or not the bursts have measured spectroscopic redshifts. If this is true for Amati relation, the results obtained based on Amati relation would be unreliable. But it is still in debating. So, before the dust settles down, we still assume that Amati relation is reliable in this paper.

This paper is structured as follows. In section 2, the values of cosmological parameter dependance in $\Lambda \mathrm{CDM}$ model are presented as shown in [18]. The errors of correlation parameters $\sigma_{a}, \sigma_{b}$ and systematic error $\sigma_{s y s}$ are also calculated via Amati relation with 109 GRBs data points. The 5 bins model-independent distance measurements will also be found in this section. In section III, we use the resulted data points to constrain $\Lambda$ CDM model as an example. A summary and discussion are put in section IV.

\section{Calibration of GRBs and Model-independent Distance Measurement}

Following the work of [3], we consider the well-known Amati $E_{p, i}-E_{i s o}$ correlation $[9,11-13]$ in GRBs, where $E_{p, i}=E_{p, o b s}(1+z)$ is the cosmological rest-frame spectral peak energy, and $E_{i s o}$ is the isotropic energy

$$
E_{\text {iso }}=4 \pi d_{L}^{2} S_{\text {bolo }} /(1+z)
$$


in which $d_{L}$ and $S_{\text {bolo }}$ are the luminosity distance and the bolometric fluence of the GRBs respectively. Following [3], we rewrite the Amati relation in the form of

$$
\log \frac{E_{i s o}}{\operatorname{erg}}=a+b \log \frac{E_{p, i}}{300 \mathrm{keV}} .
$$

One fitts the Amati relation through the minimization of $\chi^{2}$ which is given by [3]

$$
\chi^{2}=\sum_{i=1}^{N} \frac{y_{i}-a-b x_{i}}{\sigma_{y, i}^{2}+b^{2} \sigma_{x, i}^{2}},
$$

where

$$
\begin{aligned}
x_{i} & =\log \frac{E_{p, i}}{300 \mathrm{keV}} \\
y_{i} & =\log \frac{E_{\text {iso }}}{\operatorname{erg}}=\log \frac{4 \pi S_{\text {bolo }, i}}{1+z}+2 \log \bar{d}_{L}
\end{aligned}
$$

where $\bar{d}_{L}$ is defined as [18]

$$
\bar{d}_{L}=H_{0}(1+z) r(z) / c,
$$

and the errors are calculated by using the error propagation law [10]:

$$
\begin{aligned}
\sigma_{x, i} & =\frac{\sigma_{E_{p, i}}}{\ln 10 E_{p, i}} \\
\sigma_{y, i} & =\frac{\sigma_{S_{\text {bolo }, i}}}{\ln 10 S_{\text {bolo }, i}} .
\end{aligned}
$$

By calculating the value of $\chi^{2}$, we find it is large and dominated by the systematic errors, and on the contrast the statistical errors on $a$ and $b$ are small. Following [3], the systematic error $\sigma_{\text {sys }}$ can be derived by required $\chi^{2}=\nu$ (the degrees of freedom). At last, the total error $\sigma_{\text {tot }}^{2}=\sigma_{\text {stat }}^{2}+\sigma_{\text {sys }}^{2}$ is obtained. It would be noticed that in our case, the best fit value of $a$ will be less $2 \log \left(c / H_{0}\right)$ than that in the definition of luminosity distance $d_{L}=(1+z) r(z)[18]$. For this definition, the value of $H_{0}$ is absorbed into the calibration of GRBs because of the lack of fixing the absolute magnitude of GRBs. Then, in our treatment the results will be $H_{0}$ free.

As shown in [18], the calibration of GRBs is cosmological model dependent, because the values of $a$ and $b$ are obtained on the basis of $\Lambda \mathrm{CDM}$ model. So the calibrated result can not be used to constrain any other cosmological models. This the so-called well-known model dependent problem or circular problem. In Tab. 1, the cosmological parameter dependence are shown. With a careful observation, one can find that the $1 \sigma$ errors bars of $a, b$ and system are almost the same which do not depend on the cosmological parameters. In this paper as suggested by Wang [18], we shall only use the systematic error and the errors of $a$ and $b$ in the case of $\Omega_{m 0}=0.27$ for $\Lambda \mathrm{CDM}$ model as the standard values. That is to say the values of $\sigma_{a}, \sigma_{b}$ and $\sigma_{\text {sys }}$ will be used in the following sections not the values of $a$ and $b$ derived from $\Lambda \mathrm{CDM}$ model.

Following the work of Wang [18], the $\chi_{G R B}^{2}$ of a cosmological model is given by

$$
\chi_{G R B}^{2}=\sum_{i=1}^{N_{G R B}} \frac{\left[\left(\log \bar{d}_{L}^{2}\right)_{i}^{d a t a}-\log \bar{d}_{L}^{2}\left(z_{i}\right)\right]^{2}}{\left[\sigma\left(\log \bar{d}_{L}^{2}\right)_{i}^{\text {data }}\right]^{2}},
$$




\begin{tabular}{c|c|c|c}
\hline \hline & $\Omega_{m 0}=0.27$ & $\Omega_{m 0}=0.2$ & $\Omega_{m 0}=0.4$ \\
\hline$a$ & $-3.392 \pm 0.0368$ & $-3.343 \pm 0.0358$ & $-3.467 \pm 0.0364$ \\
$b$ & $1.583 \pm 0.0729$ & $1.600 \pm 0.0744$ & $1.554 \pm 0.0725$ \\
$\sigma_{\text {sys }}$ & 0.324 & 0.328 & 0.321 \\
\hline \hline
\end{tabular}

Table 1. Systematic error and values of $a$ and $b$ for GRBs Amati relation in the cases of $\Omega_{m 0}=$ $0.27,0.2,0.4$ in $\Lambda$ CDM model.

where

$$
\begin{aligned}
{\left[\sigma\left(\log \vec{d}_{L}^{2}\right)_{i}^{\text {data }}\right]^{2} } & =\sigma_{a}^{2}+\left(\sigma_{b} \log \frac{E_{p, i}}{300 \mathrm{keV}}\right)^{2}+\left(b \frac{\sigma_{E_{p, i}}}{\ln 10 E_{p, i}}\right)^{2}+\left(\frac{\sigma_{S_{\text {bolo }, i}}}{\ln 10 S_{\text {bolo }, i}}\right)^{2}+\sigma_{\text {sy }}^{2}(2,10 \\
\left(\log \vec{d}_{L}^{2}\right)_{i}^{\text {data }} & =a+b \log \frac{E_{p, i}}{300 \mathrm{keV}}-\log \frac{4 \pi S_{\text {bolo }, i}}{1+z}
\end{aligned}
$$

To constrain a cosmological model, one uses a set of model-independent distance measurements $\left\{\bar{r}_{p}\left(z_{i}\right)\right\}$ :

$$
\bar{r}_{p}\left(z_{i}\right) \equiv \frac{r_{p}(z)}{r_{p}\left(z_{0}\right)}, \quad r_{p}(z) \equiv \frac{(1+z)^{1 / 2}}{z} \frac{H_{0}}{c} r(z)
$$

where $r(z)=d_{L}(z) /(1+z)$ is the comoving distance at redshift $z, z_{0}$ is the lowest GRBs redshift ${ }^{1}$. Here, the definition of $r_{p}$ is different from Wang's definition [18] $r_{p}(z) \equiv(1+$ $z)^{1 / 2} H_{0} r(z) /(z c h)$ where $h=H_{0} /\left(100 \mathrm{kms}^{-1} \mathrm{Mpc}^{-1}\right)$. In our definition, the distance measurement $\bar{r}_{p}\left(z_{i}\right)$ and $r_{p}(z)$ is completely $H_{0}$ free. It can be seen from the facts that the definition of $r(z)$ is $r(z)=c / H_{0} \int_{0}^{z} d z^{\prime} / E\left(z^{\prime}\right)$ where $H^{2}(z)=H_{0}^{2} E^{2}(z)$ is the Hubble parameter. In terms of our definition, $\bar{d}_{L}$ can be rewritten as

$$
\bar{d}_{L}=z(1+z)^{1 / 2} r_{p}\left(z_{0}\right) \bar{r}_{p}(z) .
$$

We divide the redshifts of GRBs into $N$ bins, i.e. $\left\{z_{i}\right\}, i=1, \ldots, N$, and assume the corresponding values of $\left\{\bar{r}_{p}\left(z_{i}\right)\right\}, i=1, \ldots, N$ which do not depend on any cosmological models. Then the values of $\bar{r}_{p}(z)$ at arbitrary redshift $z$ can be obtained by cubic spline interpolation from $\left\{\bar{r}_{p}\left(z_{i}\right)\right\}$. So, the values of $d_{L}(z)$ and $\bar{d}_{L}(z)$, etc at redshift $z$ can be found easily. Given each set of $\left\{\bar{r}_{p}\left(z_{i}\right)\right\}, i=1, \ldots, N$, we calibrate the GRBs and calculate the likelihood simultaneously via Eq. (2.9) by using Markov Chain Monte Carlo (MCMC) method [19]. The MCMC is a global fitting method which is used to determine the cosmological parameters. In adopting the MCMC approach, we generate using Monte Carlo method a chain of sample points distributed in the parameter space according to the posterior probability, using the Metropolis-Hastings algorithm with uniform prior probability distribution. In the parameter space formed by the constraint cosmological parameters, a random set of initial values of the model parameters is chosen to calculate the $\chi^{2}$ or the likelihood. Whether the set of parameters can be accepted as an effective Markov chain or not is determined by the Metropolis-Hastings algorithm. The accepted set not only forms a Markov chain, but also provides a starting point for the next process. We then repeat this process until the

\footnotetext{
${ }^{1} z_{0}=0.17$ was used in [18]. In this work, the lowest redshift of GRBs is $z_{0}=0.0331$
} 
established convergence accuracy can be satisfied. The convergence is tested by checking the so-called worst e-values [the variance(mean)/mean(variance) of $1 / 2$ chains] $R-1<0.005$ [19]. As results, we obtain a set of distances $\left\{\bar{r}_{p}\left(z_{i}\right)\right\}, i=1, \ldots, N$ which are independent on any assumption of cosmological parameters. It comes from the observations that, in the process of calibration, the statistical and systematic errors of $\sigma_{a}, \sigma_{b}$ and $\sigma_{s y s}$ are only used. We $d o$ not use any values of $a$ and $b$ calibrated in $\Lambda \mathrm{CDM}$ model. The important thing is that the statistical and systematic errors $\sigma_{a}, \sigma_{b}$ and $\sigma_{s y s}$ are almost model parameter independent. Thanks to this feature, this method is model-independent. Here, in the MCMC analysis, we take $a, b$ and $N\left\{\bar{r}_{p}\left(z_{i}\right)\right\}, i=1, \ldots, N$ as free parameters. So, the degree of freedoms is $\nu=109-2-N$. Once these values of $\left\{\bar{r}_{p}\left(z_{i}\right)\right\}, i=1, \ldots, N$ are obtained, a cosmological model can be constrained by GRBs via the $\chi^{2}$

$$
\begin{aligned}
\chi_{G R B}^{2} & =\left[\Delta \bar{r}_{p}\left(z_{i}\right)\right] \cdot\left(\operatorname{Cov}_{G R B}^{-1}\right)_{i j} \cdot\left[\Delta \bar{r}_{p}\left(z_{i}\right)\right], \\
\Delta \bar{r}_{p}\left(z_{i}\right) & =\bar{r}_{p}^{\text {data }}\left(z_{i}\right)-\bar{r}_{p}\left(z_{i}\right),
\end{aligned}
$$

where $\bar{r}_{p}\left(z_{i}\right)$ is defined by Eq. (2.13) and $\left(\operatorname{Cov}_{G R B}^{-1}\right)_{i j}, i, j=1 \ldots N$ is the covariance matrix. In this way, the constraints from larger observational GRBs data points are projected into relative smaller number of points. Of course, this method can be generalized to discuss other problems.

Now, we present some discussion about the treatment of $r_{p}\left(z_{0}\right)$. Here, we do not calculate the value of $r_{p}$ at the redshift $z_{0}$ via its definition (2.13) according to any cosmological model. Because, if we calculate the value for any cosmological model, a model or cosmological parameter dependence will be introduced again. The values of $r_{p}\left(z_{0}\right)$ are fixed by the calibration relation $(2.2)$, i.e. via the relation

$$
r_{p}\left(z_{0}\right)=10^{a / 2}\left(\frac{E_{p, i}\left(z_{0}\right)}{300}\right)^{b / 2} \frac{1}{z_{0}\left(4 \pi S_{\text {bolo }}\left(z_{0}\right)\right)^{1 / 2}} .
$$

In fact, once this relation is used, one can recast Eq. (2.9) into the following form

$$
\chi_{G R B}^{2}=\sum_{i=1}^{N_{G R B}} \frac{\left(Y_{i}-Y_{0}\right)^{2}}{\left(\sigma_{Y, i}^{\text {data }}\right)^{2}},
$$

where $Y_{i}$ is defined as

$$
Y_{i}=b \log E_{p, i}\left(z_{i}\right)-\log S_{b o l o}\left(z_{i}\right)-2 \log z_{i}-2 \log \bar{r}_{p}\left(z_{i}\right)
$$

and $Y_{0}=Y_{i}\left(z_{0}\right)$, the $\sigma_{Y, i}^{\text {data }}$ is the total $1 \sigma$ errors of data sets

$$
\sigma_{\text {tot }, i}^{\text {data }}=\left(\sigma_{b} \log \frac{E_{p, i}}{300 \mathrm{keV}}\right)^{2}+\left(b \frac{\sigma_{E_{p, i}}}{\ln 10 E_{p, i}}\right)^{2}+\left(\frac{\sigma_{S_{\text {bolo }, i}}}{\ln 10 S_{\text {bolo }, i}}\right)^{2}+\sigma_{\text {sys }}^{2} .
$$

One can see that the parameter $a$ is removed from this new form of $\chi_{G R B}^{2}$. In other words, the information about the slope $b$ of GRBs correlation is used alone. So the absolute magnitude $a$ of GRBs is irrelevant in this method. Of cause, the $r_{p}\left(z_{0}\right)$ can be fixed by consulting a special cosmological model or other data sets. But in that way, the circular problem and data sets relevance problem will come back. Equivalently, the absolute magnitude of GRBs was fixed in a special cosmological model. So, for every possible values of $b$ in every running of MCMC, the $\chi_{G R B}^{2}$ is calculated. Here, we must keep in mind that the values of parameter 
$b$ are not taken from Tab. 1. It is treated as a free parameter. So, this method is completely cosmological parameter in-dependent and self consistent.

We divide the redshifts into $N=5$ bins and run the MCMC codes which is based on the publicly available CosmoMC package [19]]. The chains have worst e-value $R-1=0.0017$ which is much smaller than 0.005 . The resulted model-independent distances and covariance matrix from 109 GRBs are shown in Tab. 2 and Eq. (2.22). As already mentioned above,

\begin{tabular}{c|c|c|c|c}
\hline \hline & $z$ & $\bar{r}_{p}^{\text {data }}(z)$ & $\sigma\left(\bar{r}_{p}(z)\right)^{+}$ & $\sigma\left(\bar{r}_{p}(z)\right)^{-}$ \\
\hline 0 & 0.0331 & 1.0000 & - & - \\
1 & 1.0000 & 0.9320 & 0.1711 & 0.1720 \\
2 & 2.0700 & 0.9180 & 0.1720 & 0.1718 \\
3 & 3.0000 & 0.7795 & 0.1630 & 0.1629 \\
4 & 4.0480 & 0.7652 & 0.1936 & 0.1939 \\
5 & 8.1000 & 1.1475 & 0.4297 & 0.4389 \\
\hline \hline
\end{tabular}

Table 2. Distances measured form 109 GRBs via Amati relation with $1 \sigma$ upper and lower uncertainties.

$z_{0}=0.0331$ is adopted in this work. The $\left\{\bar{r}_{p}\left(z_{i}\right)\right\}, i=1, \ldots, 5$ correlation matrix is given by

$$
\left(\overline{\operatorname{Cov}}_{G R B}\right)=\left(\begin{array}{lllll}
1.0000 & 0.7780 & 0.8095 & 0.6777 & 0.4661 \\
0.7780 & 1.0000 & 0.7260 & 0.6712 & 0.3880 \\
0.8095 & 0.7260 & 1.0000 & 0.6046 & 0.5032 \\
0.6777 & 0.6712 & 0.6046 & 1.0000 & 0.1557 \\
0.4661 & 0.3880 & 0.5032 & 0.1557 & 1.0000
\end{array}\right)
$$

and the corresponding covariance matrix is given by

$$
\left(\operatorname{Cov}_{G R B}\right)_{i j}=\sigma\left(\bar{r}_{p}\left(z_{i}\right)\right) \sigma\left(\bar{r}_{p}\left(z_{j}\right)\right)\left(\overline{\operatorname{Cov}}_{G R B}\right)_{i j},
$$

where

$$
\begin{array}{ccc}
\sigma\left(\bar{r}_{p}\left(z_{i}\right)\right)=\sigma\left(\bar{r}_{p}\left(z_{i}\right)\right)^{+}, & \text {if } \quad \bar{r}_{p}(z) \geq \bar{r}_{p}(z)^{\text {data }} \\
\sigma\left(\bar{r}_{p}\left(z_{i}\right)\right)=\sigma\left(\bar{r}_{p}\left(z_{i}\right)\right)^{-}, & \text {if } \quad & \bar{r}_{p}(z)<\bar{r}_{p}(z)^{\text {data }}
\end{array}
$$

here the $\sigma\left(\bar{r}_{p}\left(z_{i}\right)\right)^{+}$and $\sigma\left(\bar{r}_{p}\left(z_{i}\right)\right)^{-}$are the $1 \sigma$ errors listed in Tab. 2. The marginalized values and the corresponding upper and lower bounds are used when GRBs is used as a cosmological constraint. The $1 D$ distributions of model parameters are shown in Fig.1. The distance measurements from 109 GRBs via Amati correlation with $1 \sigma$ error bars are shown in Fig. 2, where the solid lines correspond to $\Lambda$ CDM model with different values of $\Omega_{m 0}=0.2,0.27,0.4$ from up to bottom respectively.

The data points shown in Tab. 2 and Fig. 2 are totally cosmological model independent, so one can use them to constrain any other cosmological models. In the next section, as an example, these obtained data points will be used to constrain $\Lambda$ CDM model. From Fig. 2, one can find that GRBs favors large values of $\Omega_{m 0}$ for $\Lambda$ CDM model. This point can be confirmed in the next section. And the concordance model $\left(\Omega_{m 0}=0.27\right)$ is almost at the boundary of $1 \sigma$ regions of data points. So the null hypothesis that Amati relation is based on cosmology can be rejected slightly greater than $4.5 \sigma$ as shown in Fig. 2. 

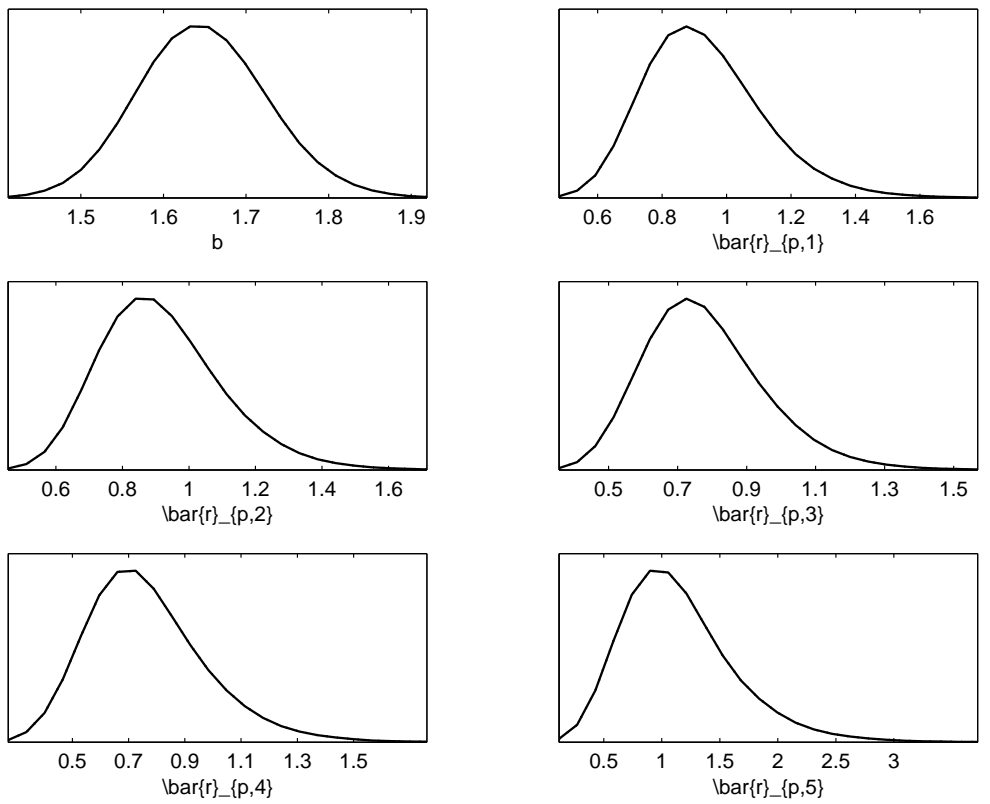

Figure 1. The $1 D$ marginalized probabilities of model parameters.

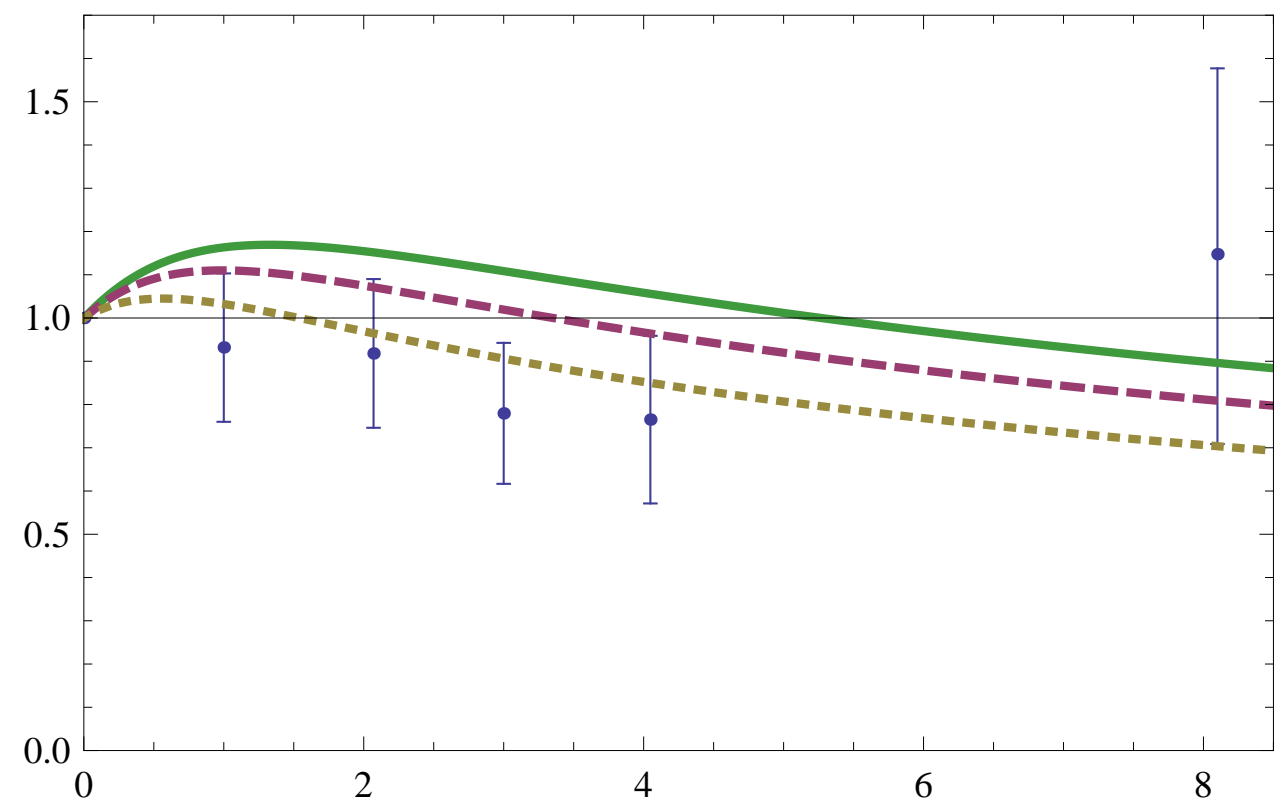

Figure 2. The mean values of distance measurement from 109 GRBs via Amati relation with $1 \sigma$ error bars. The lines from up to bottom correspond to $\Omega_{m 0}=0.2,0.27,0.4$ respectively. 


\section{Cosmological Constraint to Dark Energy}

In our calculations, we have taken the total likelihood function $L \propto e^{-\chi^{2} / 2}$ to be the products of the separate likelihoods of SN, BAO, CMB and GRBs. Then we get $\chi^{2}$

$$
\chi^{2}=\chi_{S N}^{2}+\chi_{B A O}^{2}+\chi_{C M B}^{2}+\chi_{G R B}^{2},
$$

where the separate likelihoods of $\mathrm{SN}, \mathrm{BAO}, \mathrm{CMB}$ are shown in the Appendix A. The $\chi_{G R B}^{2}$ is the form of Eq. (2.15). In this work, we only consider the $\Lambda$ CDM model as a simple example. Its generalization to constrain other cosmological models is straight forward. In the case, when SN Ia and GRBs are combined as cosmic constraint, we have $\chi_{\min }^{2}=547.727$ and the best fit values of model parameter $\Omega_{m 0}=0.274_{-0.0194}^{+0.0203}$. If the SN Ia is used alone, one has $\chi_{\min }^{2}=542.680$ and $\Omega_{m 0}=0.270_{-0.0213}^{+0.0222}$. The corresponding contour plot is shown in Fig. 3. When combing the cosmic observations considered in this work together, we

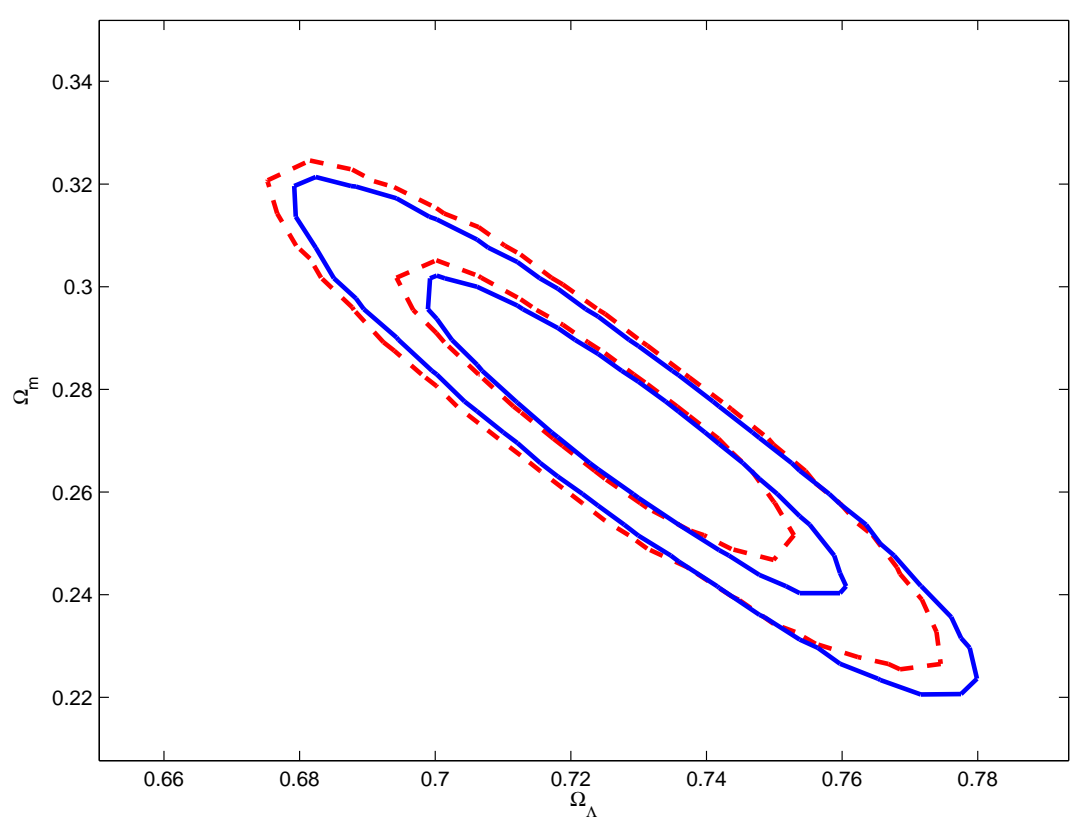

Figure 3. Color Online: $2 D$ contours plots in the $\Omega_{\Lambda}-\Omega_{m}$ plane. The solid blue lines denote the $1 \sigma$ and $2 \sigma$ regions from SN Union 2 alone. The red dashed lines denote the $1 \sigma$ and $2 \sigma$ regions from the combination of SN Union 2 and 109 GRBs.

have the resulted $\chi_{\min }^{2}=549.383$ and $\Omega_{m 0}=0.279_{-0.0131}^{+0.0138}$. Also, the best fit values of $\Omega_{m 0}=0.277_{-0.0128}^{+0.0132}$ and $\chi_{\min }^{2}=544.451$ when GRBs is not used for comparison. The $2 D$ contour plots are shown in Fig. 4. We can find that when GRBs is used, the relative errors of model parameters is shrunken. Here, we can compare our result with Wei's one where he used 50 low redshift GRBs to calibrate the Amati relation. So, we add 59 high redshift data points of GRBs to SN Union 2 data sets. After constraint via MCMC, we have $\chi_{\min }^{2}=565.919$ and $\Omega_{m 0}=0.271_{-0.0192}^{+0.0198}$. The corresponding $2 D$ contour plot is shown in Fig. 5. The constrained results show that relative large values of $\Omega_{m 0}$ are favored when GRBs data points are employed. This is consistent with the clues shown in Fig. 2. 


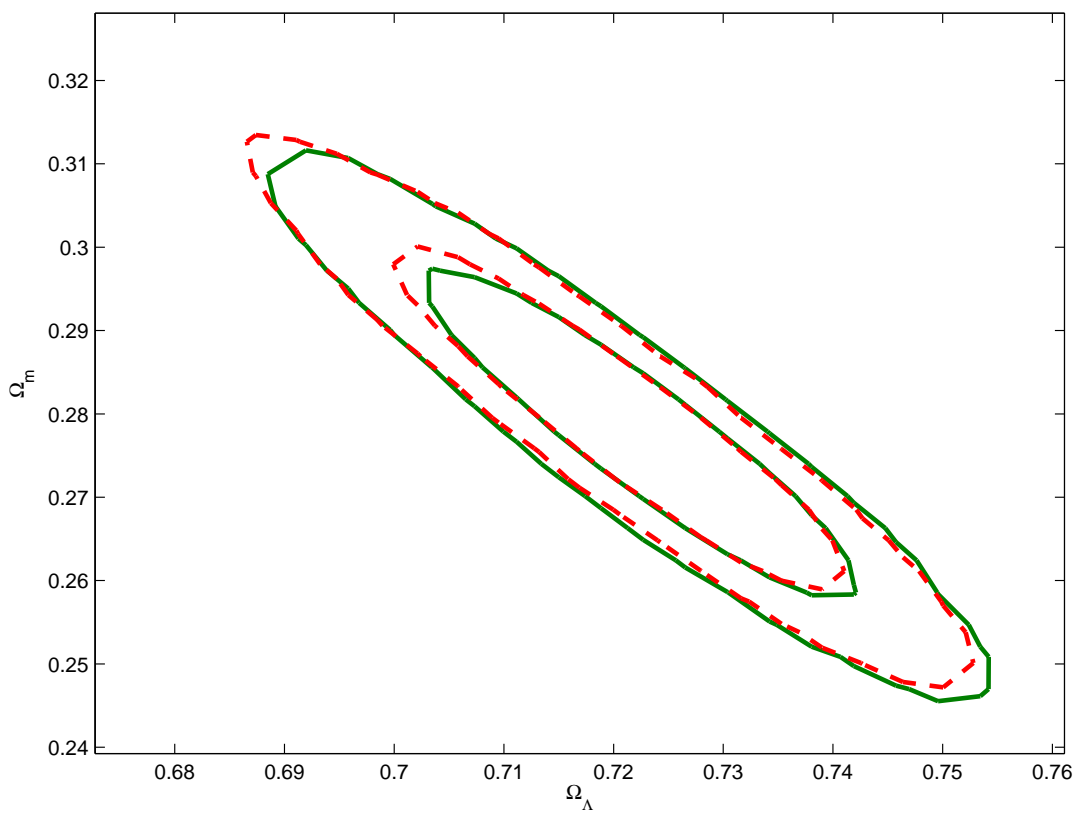

Figure 4. Color Online: $2 D$ contours plots in the $\Omega_{\Lambda}-\Omega_{m}$ plane. The solid blue lines denote the $1 \sigma$ and $2 \sigma$ regions from $\mathrm{SN}+\mathrm{BAO}+\mathrm{CMB}$. The red dashed lines denote the $1 \sigma$ and $2 \sigma$ regions from the combination of $\mathrm{SN}+\mathrm{BAO}+\mathrm{CMB}+\mathrm{GRBs}$.

\section{Summary and Discussion}

In this paper, by using 109 GRBs data points via Amati relation, we have derived five data points of distance measurements which do not depend on any cosmological models, i.e. in a model-independent way, based on the method firstly advocated by Wang [18]. Then it can be used to constrain any other cosmological models without the so-called circular and data sets consistence problem. We also find out that the concordance model $\left(\Omega_{m 0}=0.27\right)$ is almost at the boundary of $1 \sigma$ regions of data points. So, one can reject the null hypothesis that Amati relation is based on cosmology slightly greater than $4.5 \sigma$ as shown in Fig. 2.

When GRBs (the five data points of distance measurements) is used as a complementary cosmic constraint to $\Lambda \mathrm{CDM}$ model as an example, the errors of model parameters are shrunken. Though the constraint is not much tighter than that calibrated via low redshift $\mathrm{SN}$, the difference is very small. And, the results can be used to constrain other cosmological model beyond $\Lambda$ CDM. The important thing is that it does not depend on SN Ia data set. The merits of this method has been mentioned in the introduction. Here, we list possible potential drawbacks of this completely model in-dependent method as follows: (i). The constraint is not much tighter than that obtained from calibration by using SN Ia data points. The looser may come from the lack of enough GRBs data points or the number of bins is not larger. (ii). How to design a cosmological model indicator, here it is model independent distance measurement $\bar{r}_{p}(z)$, which is sensitive to distinguish the cosmological models may be the main points of this method. In this way, the lack of having no enough GRBs data points can be avoided in some senses. (iii) Another negative effect comes from the choice of fiducial redshift $z_{0}$. Some average values of $r_{p}\left(z_{0}\right)$ would be better. 


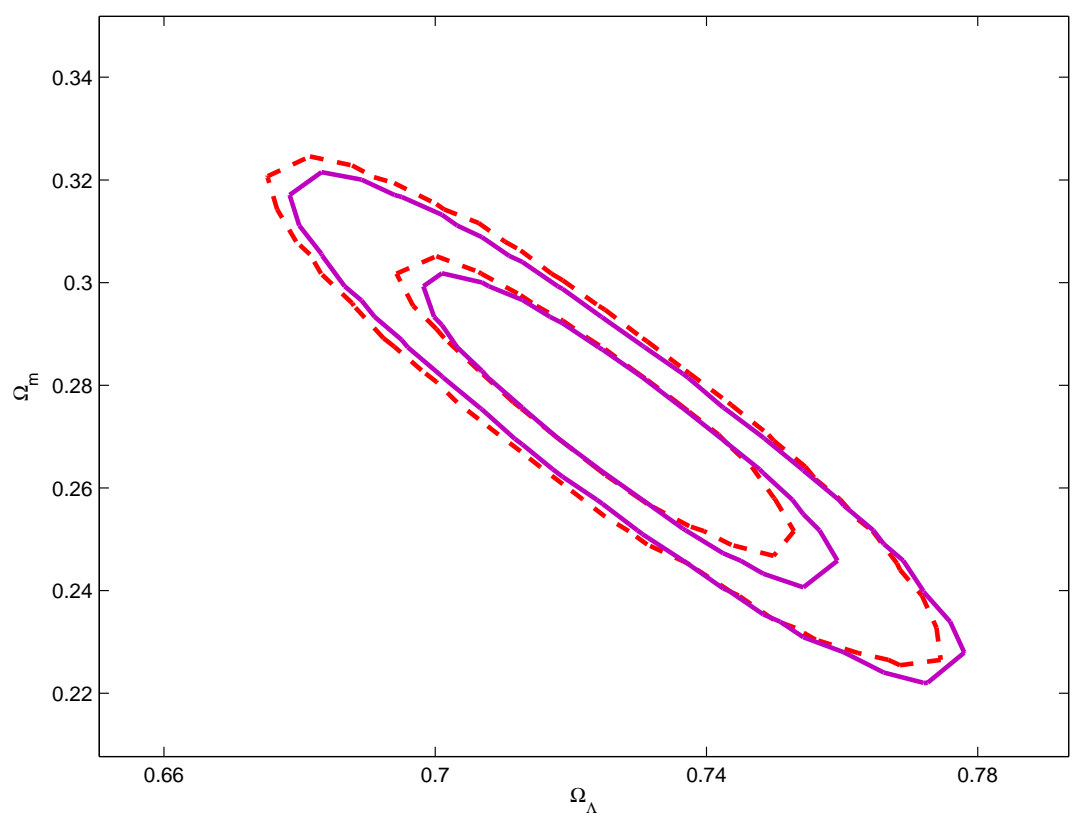

Figure 5. Color Online: $2 D$ contours plots in the $\Omega_{\Lambda}-\Omega_{m}$ plane. The solid pink lines denote the $1 \sigma$ and $2 \sigma$ regions from SN Union $2+59$ GRBs from Wei's results. The red dashed lines denote the $1 \sigma$ and $2 \sigma$ regions from the combination of $\mathrm{SN}+\mathrm{GRBs}$ in our case.

At last we have to alert the readers that our analysis is based on the assumption that Amati relation is reliable. However, in the literatures, the Amati relation has been criticized for many reasons as mentioned in the introduction. If that is true, our analysis would be unreliable.

\section{Acknowledgments}

The author thanks an anonymous referee for invaluable improvement of this paper and Dr. Jirong Mao for useful discussion on Amati relation. This work is supported by the Fundamental Research Funds for the Central Universities (DUT10LK31) and (DUT11LK39) of P.R. China.

\section{A Cosmic Observations: SN, BAO and CMB}

\section{A.1 Type Ia Supernovae constraints}

Recently, SCP (Supernova Cosmology Project) collaboration released their Union2 dataset which consists of $557 \mathrm{SN}$ Ia [21]. The distance modulus $\mu(z)$ is defined as

$$
\mu_{t h}(z)=5 \log _{10}\left[\bar{d}_{L}(z)\right]+\mu_{0},
$$

where $\bar{d}_{L}(z)$ is the Hubble-free luminosity distance $H_{0} d_{L}(z) / c=H_{0} d_{A}(z)(1+z)^{2} / c$, with $H_{0}$ the Hubble constant, defined through the re-normalized quantity $h$ as $H_{0}=100 h \mathrm{~km} \mathrm{~s}^{-1} \mathrm{Mpc}^{-1}$, 
and $\mu_{0} \equiv 42.38-5 \log _{10} h$. Additionally, the observed distance moduli $\mu_{o b s}\left(z_{i}\right)$ of SN Ia at $z_{i}$ is

$$
\mu_{o b s}\left(z_{i}\right)=m_{o b s}\left(z_{i}\right)-M,
$$

where $M$ is their absolute magnitudes.

For the SN Ia dataset, the best fit values of the parameters $p_{s}$ can be determined by a likelihood analysis, based on the calculation of

$$
\begin{aligned}
\chi^{2}\left(p_{s}, M^{\prime}\right) & \equiv \sum_{S N} \frac{\left\{\mu_{o b s}\left(z_{i}\right)-\mu_{t h}\left(p_{s}, z_{i}\right)\right\}^{2}}{\sigma_{i}^{2}} \\
& =\sum_{S N} \frac{\left\{5 \log _{10}\left[\bar{d}_{L}\left(p_{s}, z_{i}\right)\right]-m_{o b s}\left(z_{i}\right)+M^{\prime}\right\}^{2}}{\sigma_{i}^{2}},
\end{aligned}
$$

where $M^{\prime} \equiv \mu_{0}+M$ is a nuisance parameter which includes the absolute magnitude and the parameter $h$. The nuisance parameter $M^{\prime}$ can be marginalized over analytically [22] as

$$
\bar{\chi}^{2}\left(p_{s}\right)=-2 \ln \int_{-\infty}^{+\infty} \exp \left[-\frac{1}{2} \chi^{2}\left(p_{s}, M^{\prime}\right)\right] d M^{\prime},
$$

resulting to

$$
\bar{\chi}^{2}=A-\frac{B^{2}}{C}+\ln \left(\frac{C}{2 \pi}\right),
$$

with

$$
\begin{aligned}
A & =\sum_{S N} \frac{\left\{5 \log _{10}\left[\bar{d}_{L}\left(p_{s}, z_{i}\right)\right]-m_{\text {obs }}\left(z_{i}\right)\right\}^{2}}{\sigma_{i}^{2}} \\
B & =\sum_{S N} \frac{5 \log _{10}\left[\bar{d}_{L}\left(p_{s}, z_{i}\right)\right]-m_{o b s}\left(z_{i}\right)}{\sigma_{i}^{2}} \\
C & =\sum_{S N} \frac{1}{\sigma_{i}^{2}} .
\end{aligned}
$$

Relation (A.3) has a minimum at the nuisance parameter value $M^{\prime}=B / C$, which contains information of the values of $h$ and $M$. Therefore, one can extract the values of $h$ and $M$ provided the knowledge of one of them. Finally, note that the expression

$$
\chi_{S N}^{2}\left(p_{s}, B / C\right)=A-\left(B^{2} / C\right),
$$

which coincides to Eq. (A.4) up to a constant, is often used in the likelihood analysis [22, 23], and thus in this case the results will not be affected by a flat $M^{\prime}$ distribution.

\section{A.2 Baryon Acoustic Oscillation constraints}

The Baryon Acoustic Oscillations are detected in the clustering of the combined the $2 \mathrm{dF}$ Galaxy Redshift Survey (2dFGRS) and Sloan Digital Sky Survey (SDSS) main galaxy samples, and measure the distance-redshift relation at $z=0.2$. Additionally, Baryon Acoustic Oscillations in the clustering of the SDSS luminous red galaxies measure the distance-redshift relation at $z=0.35$. The observed scale of the BAO calculated from these samples, as well 
as from the combined sample, are jointly analyzed using estimates of the correlated errors to constrain the form of the distance measurement $D_{V}(z)[24-26]$

$$
D_{V}(z)=c\left(\frac{z}{\Omega_{k} H(z)} \operatorname{sinn}^{2}\left[\sqrt{\left|\Omega_{k}\right|} \int_{0}^{z} \frac{d z^{\prime}}{H\left(z^{\prime}\right)}\right]\right)^{1 / 3} .
$$

where $\operatorname{sinn}(x)=\sin (x), x, \sinh (x)$ for $\Omega_{k}<0, \Omega_{k}=0$ and $\Omega_{k}>0$ respectively. The peak positions of the BAO depend on the ratio of $D_{V}(z)$ to the sound horizon size at the drag epoch (where baryons were released from photons) $z_{d}$, which can be obtained by using a fitting formula [27]:

$$
z_{d}=\frac{1291\left(\Omega_{m} h^{2}\right)^{-0.419}}{1+0.659\left(\Omega_{m} h^{2}\right)^{0.828}}\left[1+b_{1}\left(\Omega_{b} h^{2}\right)^{b_{2}}\right]
$$

with

$$
\begin{aligned}
& b_{1}=0.313\left(\Omega_{m} h^{2}\right)^{-0.419}\left[1+0.607\left(\Omega_{m} h^{2}\right)^{0.674}\right], \\
& b_{2}=0.238\left(\Omega_{m} h^{2}\right)^{0.223} .
\end{aligned}
$$

In this paper, we use the data of $r_{s}\left(z_{d}\right) / D_{V}(z)$ extracted from the Sloan Digitial Sky Survey (SDSS) and the Two Degree Field Galaxy Redshift Survey (2dFGRS) [28], which are listed in Table 3, where $r_{s}(z)$ is the comoving sound horizon size

$$
\begin{aligned}
r_{s}(z) & =c \int_{0}^{t} \frac{c_{s} d t}{a}=c \int_{0}^{a} \frac{c_{s} d a}{a^{2} H}=c \int_{z}^{\infty} d z \frac{c_{s}}{H(z)} \\
& =\frac{c}{\sqrt{3}} \int_{0}^{1 /(1+z)} \frac{d a}{a^{2} H(a) \sqrt{1+\left(3 \Omega_{b} /\left(4 \Omega_{\gamma}\right) a\right)}},
\end{aligned}
$$

where $c_{s}$ is the sound speed of the photon-baryon fluid [29-31]:

$$
c_{s}^{-2}=3+\frac{4}{3} \times \frac{\rho_{b}(z)}{\rho_{\gamma}(z)}=3+\frac{4}{3} \times\left(\frac{\Omega_{b}}{\Omega_{\gamma}}\right) a,
$$

and here $\Omega_{\gamma}=2.469 \times 10^{-5} h^{-2}$ for $T_{C M B}=2.75 K$.

\begin{tabular}{c|c}
\hline \hline$z$ & $r_{s}\left(z_{d}\right) / D_{V}(z)$ \\
\hline 0.2 & $0.1905 \pm 0.0061$ \\
\hline 0.35 & $0.1097 \pm 0.0036$ \\
\hline
\end{tabular}

Table 3. The observational $r_{s}\left(z_{d}\right) / D_{V}(z)$ data [25].

Using the data of $\mathrm{BAO}$ in Table 3 and the inverse covariance matrix $V^{-1}$ in [25]:

$$
V^{-1}=\left(\begin{array}{cc}
30124.1 & -17226.9 \\
-17226.9 & 86976.6
\end{array}\right)
$$

Thus, the $\chi_{B A O}^{2}\left(p_{s}\right)$ is given as

$$
\chi_{B A O}^{2}\left(p_{s}\right)=X^{t} V^{-1} X,
$$


where $X$ is a column vector formed from the values of theory minus the corresponding observational data, with

$$
X=\left(\begin{array}{c}
\frac{r_{s}\left(z_{d}\right)}{D_{V}(0.2)}-0.190533 \\
\frac{r_{s}\left(z_{d}\right)}{D_{V}(0.35)}-0.109715
\end{array}\right),
$$

and $X^{t}$ denotes its transpose.

\section{A.3 Cosmic Microwave Background constraints}

The CMB shift parameter $R$ is provided by [32]

$$
R\left(z_{*}\right)=\frac{\sqrt{\Omega_{m} H_{0}^{2}}}{\sqrt{\left|\Omega_{k}\right|}} \operatorname{sinn}\left[\sqrt{\left|\Omega_{k}\right|} \int_{0}^{z_{*}} \frac{d z^{\prime}}{H\left(z^{\prime}\right)}\right]
$$

here, the redshift $z_{*}$ (the decoupling epoch of photons) is obtained using the fitting function [33]

$$
z_{*}=1048\left[1+0.00124\left(\Omega_{b} h^{2}\right)^{-0.738}\right]\left[1+g_{1}\left(\Omega_{m} h^{2}\right)^{g_{2}}\right],
$$

where the functions $g_{1}$ and $g_{2}$ read

$$
\begin{aligned}
& g_{1}=0.0783\left(\Omega_{b} h^{2}\right)^{-0.238}\left(1+39.5\left(\Omega_{b} h^{2}\right)^{0.763}\right)^{-1}, \\
& g_{2}=0.560\left(1+21.1\left(\Omega_{b} h^{2}\right)^{1.81}\right)^{-1} .
\end{aligned}
$$

In additional, the acoustic scale is related to the first distance ratio and is expressed as

$$
l_{A}=\frac{\pi}{r_{s}\left(z_{*}\right)} \frac{c}{\sqrt{\left|\Omega_{k}\right|}} \operatorname{sinn}\left[\sqrt{\left|\Omega_{k}\right|} \int_{0}^{z_{*}} \frac{d z^{\prime}}{H\left(z^{\prime}\right)}\right] .
$$

\begin{tabular}{c|ccc}
\hline \hline & $7-$ year ML & 7 - year mean & error, $\sigma$ \\
\hline$l_{A}\left(z_{*}\right)$ & 302.09 & 302.69 & 0.76 \\
\hline$R\left(z_{*}\right)$ & 1.725 & 1.726 & 0.018 \\
\hline$z_{*}$ & 1091.3 & 1091.36 & 0.91 \\
\hline
\end{tabular}

Table 4. The observational $l_{A}, R, z_{*}$ data [34]. The ML values are used in this work as recommended.

Using the data of $l_{A}, R, z_{*}$ in [34], which are listed in Table 4, and their covariance matrix of $\left[l_{A}\left(z_{*}\right), R\left(z_{*}\right), z_{*}\right]$ referring to [34]:

$$
C^{-1}=\left(\begin{array}{ccc}
2.305 & 29.698 & -1.333 \\
29.698 & 6825.270 & -113.180 \\
-1.333 & -113.180 & 3.414
\end{array}\right),
$$

we can calculate the likelihood $L$ as $\chi_{C M B}^{2}=-2 \ln L$ :

$$
\chi_{C M B}^{2}=\triangle d_{i}\left[C^{-1}\left(d_{i}, d_{j}\right)\right]\left[\triangle d_{i}\right]^{t},
$$

where $\triangle d_{i}=d_{i}-d_{i}^{\text {data }}$ is a row vector, and $d_{i}=\left(l_{A}, R, z_{*}\right)$. 


\section{References}

[1] A. G. Riess, et al., Astron. J. 116 1009(1998) [astro-ph/9805201].

[2] S. Perlmutter, et al., Astrophys. J. 517 565(1999) [astro-ph/9812133].

[3] B. E. Schaefer, Astrophys. J. 660, 16 (2007) [astro-ph/0612285].

[4] H. Li, J. Q. Xia, J. Liu, G. B. Zhao, Z. H. Fan, X. Zhang, Astrophys. J. 680, 92(2008).

[5] S. Capozziello, L. Izzo, Astron. Astrophys, 490, 31( 2008); V. Vitagliano, J. Q. Xia, S. Liberati, M. Viel, JCAP03(2010)005.

[6] N. Liang, W. K. Xiao, Y. Liu and S. N. Zhang, Astrophys. J. 685, 354 (2008) [arXiv:0802.4262]; N. Liang and S. N. Zhang, AIP Conf. Proc. 1065, 367 (2008) [arXiv:0808.2655]; T. S. Wang and N. Liang, arXiv:0910.5835 [astro-ph.CO]; N. Liang, P. Wu and S. N. Zhang, Phys. Rev. D 81, 083518 (2010) [arXiv:0911.5644]; H. Gao, N. Liang and Z. H. Zhu, arXiv:1003.5755 [astro-ph.CO].

[7] H. Wei and S. N. Zhang, Eur. Phys. J. C 63, 139 (2009) [arXiv:0808.2240];

[8] H. Wei, JCAP1008:020(2010), arXiv:1004.4951 [astro-ph.CO].

[9] L. Amati et al., Astron. Astrophys. 390, 81 (2002) [astro-ph/0205230].

[10] Herman J. Mosquera Cuesta, Habib Dumet M., Cristina Furlanetto, JCAP0807,004(2008).

[11] L. Amati et al., Mon. Not. Roy. Astron. Soc. 391, 577 (2008) [arXiv:0805.0377].

[12] L. Amati, arXiv:1002.2232 [astro-ph.HE]; L. Amati, Mon. Not. Roy. Astron. Soc. 372, 233 (2006) [astro-ph/0601553].

[13] L. Amati, F. Frontera and C. Guidorzi, arXiv:0907.0384 [astro-ph.HE].

[14] L.-X. Li, Mon.Not.Roy.Astron.Soc., Lett, 374, L20(2006).

[15] E. Nakar, T. Piran, Mon.Not.Roy.Astron.Soc., Lett, 360, L73(2005).

[16] D. L. Band, R.D. Preece, ApJ, 627, 319(2005).

[17] A. C. Collazzi, B. E. Schaefer, A. Goldstein, R.D. Preece, arXiv:1112.4347[astro-ph.HE].

[18] Y. Wang, Phys.Rev.D 78,123532(2008).

[19] http://cosmologist.info/cosmomc/; A. Lewis and S. Bridle, Phys. Rev. D 66, 103511 (2002).

[20] W. M. Wood-Vasey et al., Astrophys. J. 666694 (2007) [astro-ph/0701041].

[21] R. Amanullah et al. [Supernova Cosmology Project Collaboration], arXiv:1004.1711 [astro-ph.CO].

[22] S. Nesseris and L. Perivolaropoulos, Phys. Rev. D 72123519 (2005); L. Perivolaropoulos, Phys. Rev. D 71063503 (2005); E. Di Pietro and J. F. Claeskens, Mon. Not. Roy. Astron. Soc. 341 1299 (2003); A. C. C. Guimaraes, J. V. Cunha and J. A. S. Lima, JCAP 0910010 (2009).

[23] E. Garcia-Berro, E. Gaztanaga, J. Isern, O. Benvenuto and L. Althaus, astro-ph/9907440; A. Riazuelo and J. Uzan, Phys. Rev. D 66023525 (2002); V. Acquaviva and L. Verde, JCAP 0712 001 (2007).

[24] T. Okumura, T. Matsubara, D. J. Eisenstein, I. Kayo, C. Hikage, A. S. Szalay and D. P. Schneider, Astrophys. J. 676889 (2008).

[25] W. J. Percival et al., arXiv:0907.1660 [astro-ph.CO].

[26] D. J. Eisenstein et al., [SDSS Collabaration], Astrophys. J. 633560 (2005) [astro-ph/0501171].

[27] D. J. Eisenstein and W. Hu, Astrophys. J. 496605 (1998).

[28] W. J. Percival et al., Mon. Not. R. Astron. Soc. 3811053 (2007) arXiv:0705.3323 [astro-ph.CO]. 
[29] W. Hu and N. Sugiyama, Astrophys. J. 444489 (1995) [arXiv:astro-ph/9407093].

[30] W. Hu, M. Fukugita, M. Zaldarriaga and M. Tegmark, Astrophys. J. 549669 (2001) [arXiv:astro-ph/0006436].

[31] R. R. Caldwell and M. Doran, Phys. Rev. D 69103517 (2004).

[32] J. R. Bond, G. Efstathiou and M. Tegmark, Mon. Not. Roy. Astron. Soc. 291 L33 (1997).

[33] W. Hu and N. Sugiyama, Astrophys. J. 471542 (1996).

[34] E. Komatsu et al. [WMAP Collaboration], arXiv:1001.4538 [astro-ph.CO]. 